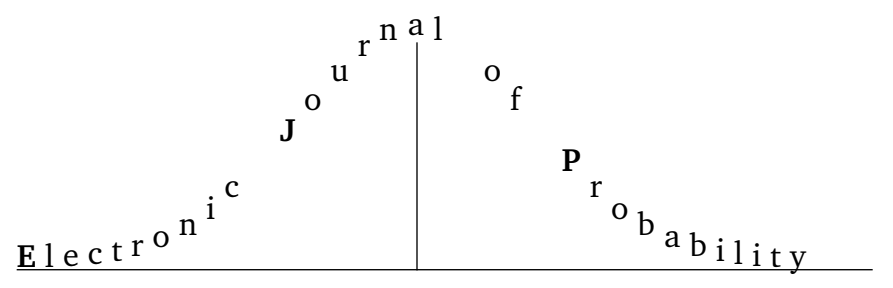

Vol. 16 (2011), Paper no. 49, pages 1341-.

Journal URL

http://www.math.washington.edu/ ejpecp/

\title{
Some sufficient conditions for infinite collisions of simple random walks on a wedge comb
}

\author{
Xinxing Chen* and Dayue Chen ${ }^{\dagger}$
}

\begin{abstract}
In this paper, we give some sufficient conditions for the infinite collisions of independent simple random walks on a wedge comb with profile $\{f(n), n \in \mathbb{Z}\}$. One interesting result is that two independent simple random walks on the wedge comb will collide infinitely many times if $f(n)$ has a growth order as $n \log n$. On the other hand, if $\{f(n), n \in \mathbb{Z}\}$ are given by i.i.d. nonnegative random variables with finite mean, then for almost all wedge combs with such profile, three independent simple random walks on it will collide infinitely many times.
\end{abstract}

Key words: wedge comb, simple random walk, infinite collision property, local time.

AMS 2010 Subject Classification: Primary 60J10, 60K37.

Submitted to EJP on November 1, 2010, final version accepted June 21, 2011.

*Department of Mathematics, Shanghai Jiaotong University, Shanghai 200240, China; Research partially supported by the NSFC grant No. 11001173. chenxinx@sjtu.edu.cn

†School of Mathematical Sciences \& Center for Statistical Science, Peking University, Beijing 100871, China; Research partially supported by the NSFC grant No. 10625101 and the 973 grant No. 2011CB808000. dayue@pku.edu.cn, http://www.math.pku.edu.cn/teachers/dayue/indexE.htm 


\section{Introduction}

In this paper, we study the number of collisions of two independent simple random walks on an infinite connected graph with finite degrees. Let $X=\left\{X_{n}\right\}$ and $X^{\prime}=\left\{X_{n}^{\prime}\right\}$ be independent simple random walks starting from the same vertex. As usual, we say that the graph has the infinite collision property if $X$ and $X^{\prime}$ collide infinitely often, i.e., $\left|\left\{n: X_{n}=X_{n}^{\prime}\right\}\right|=\infty$, almost surely. Likewise we say that the graph has the finite collision property if $X$ and $X^{\prime}$ collide finitely many times almost surely. Krishnapur and Peres [5] first finds the example $\operatorname{Comb}(\mathbb{Z})$ on which two independent simple random walks will collide finitely many times. Notice that $\mathbb{Z} \subset \operatorname{Comb}(\mathbb{Z}) \subset \mathbb{Z}^{2}$ and both $\mathbb{Z}$ and $\mathbb{Z}^{2}$ have the infinite collision property. This indicates that the infinite collision property is not simply monotone. So we are interested in probing the subgraphs of $\operatorname{Comb}(\mathbb{Z})$.

Let $f$ be a function mapping $\mathbb{Z}$ into $\mathbb{R}^{+}$. It induces a graph called wedge comb, $\operatorname{Comb}(\mathbb{Z}, f)$, with the set of vertices

$$
\mathbb{V}=\{(n, x): n, x \in \mathbb{Z},-f(n) \leq x \leq f(n)\}
$$

and the set of edges $\{[(n, x),(n, y)]:|x-y|=1\} \cup\{[(n, 0),(m, 0)]:|m-n|=1\}$.

Chen, Wei and Zhang [3] shows that $\operatorname{Comb}(\mathbb{Z}, f)$ has the infinite collision property when $f(n) \leq n^{\frac{1}{5}}$. Recently, Barlow, Peres and Sousi [1] gives a sufficient condition (in terms of Green functions) for the infinite collision property and shows that $\operatorname{Comb}(\mathbb{Z}, f)$ has the infinite collision property when $f(n) \leq n$; while it has the finite collision property when $f(n)=n^{\alpha}$ for each $\alpha>1$. Collisions on other graphes, such as random clusters and random trees are studied in [1] and [2]. We will restrict our attention to wedge combs, and give a sufficient condition for a wedge comb to have the infinite collision property.

Theorem 1.1. Let $\breve{f}(n)=1 \vee \max _{-n \leq i \leq n} f(i)$. If

$$
\sum_{n=1}^{\infty} \frac{1}{\breve{f}(n)}=\infty,
$$

then $\operatorname{Comb}(\mathbb{Z}, f)$ has the infinite collision property.

We like to point out that it is not required that $f(n)$ is monotone in $n$ for $n \geq 0$. This would enable us to deal with a large class of wedge combs. As an application of Theorem 1.1, one can improve the results of [3] and [1].

Corollary 1.2. If $f(-n)+f(n)=O(n \log n)$, then $\operatorname{Comb}(\mathbb{Z}, f)$ has the infinite collision property.

Corollary 1.2 should be compared with Theorem 1.3 . Unfortunately there is a gap. We still do not know the answer for $1<\beta \leq 2$.

Theorem 1.3. Let $f(x)=|x| \log ^{\beta}(|x| \vee 1)$ for $x \in \mathbb{Z}$. If $\beta>2$, then the total number of collisions by two independent simple random walks on $\operatorname{Comb}(\mathbb{Z}, f)$ is a.s. finite.

A natural question to ask is what happens if there are more than two independent simple random walks. Suppose that $X, X^{\prime}$ and $X^{\prime \prime}$ are independent simple random walks on a graph. If $\mid\left\{n: X_{n}=\right.$ $\left.X_{n}^{\prime}=X_{n}^{\prime \prime}\right\} \mid=\infty$ almost surely, then we say that the three processes collide together infinitely often and that the graph has the infinitely many triple collisions property. It is shown in [1] that $\mathbb{Z}$ has the infinitely many triple collisions property; while $\operatorname{Comb}(\mathbb{Z}, \alpha)$ has the finitely many triple collisions property for any $\alpha>0$. 
Theorem 1.4. Let $\{f(n), n \in \mathbb{Z}\}$ are independent and identically distributed random variables with law $\mu$ supported in $[0, \infty)$. If $\mu$ has finite mean, then almost surely $\operatorname{Comb}(\mathbb{Z}, f)$ has the infinitely many triple collisions property.

Theorem 1.1 will be proved in the next section. Proofs of Theorems 1.3 and 1.4 will be given in Section 4 and Section 3 respectively. Throughout this paper we use the following notation. For $u, v \in \mathbb{V}, \mathbf{P}^{u}$ is the probability measure of the simple random walk $X$ starting from $u$, and $\mathbf{P}^{u, v}$ is the joint probability measure of the two random walks $X$ and $X^{\prime}$ starting from $u$ and $v$, respectively. $\mathbf{E}^{u}$ and $\mathbf{E}^{u, v}$ stand for the corresponding expectations. For vertex $x \in \mathbb{V}$, we write $x_{1}$ for the first coordinate of $x$ and $x_{2}$ for the second coordinate, i.e., $x=\left(x_{1}, x_{2}\right)$. For $a, b \in \mathbb{R}, a \vee b=\max \{a, b\}$ and $a \wedge b=\min \{a, b\}$. For set $A,|A|$ stands for the number of elements of $A$.

\section{Proof of Theorem 1.1}

Let $X=\left\{X_{n}, n \geq 0\right\}$ be a simple random walk on $\operatorname{Comb}(\mathbb{Z}, f)$. Write $X_{n}=\left(U_{n}, V_{n}\right)$ for $n \geq 0$. Then $\left\{U_{n}\right\}$ is a random process on $\mathbb{Z}$. Define a sequence of stopping times: $T_{0}=0$ and

$$
T_{k+1}:=\inf \left\{n>T_{k}: U_{n} \neq U_{n-1}\right\} .
$$

Almost surely $T_{k}<\infty$ for each $k \geq 1$. By definition, $X$ stays in the line segment $\{u\} \times[-f(u), f(u)]$, where $u=U_{T_{k}}$, during time $\left[T_{k}, T_{k+1}-1\right]$. Let $W_{k}=U_{T_{k}}$. Then $\left\{W_{k}, k \geq 0\right\}$ is a simple random walk on $\mathbb{Z}$ (by the strong Markov property).

For $n \geq 1$, let

$$
\mathbb{V}_{n}=\left\{\left(x_{1}, x_{2}\right) \in \mathbb{V}:\left|x_{1}\right| \leq n\right\}
$$

and

$$
\theta_{n}=\inf \left\{m \geq 0: X_{m} \notin \mathbb{V}_{n-1}\right\} .
$$

Notice that if $X_{0} \in \mathbb{V}_{n-1}$ then $\theta_{n}$ is the hitting time of $\{(-n, 0),(n, 0)\}$ by $X$.

Let $X^{\prime}$ be another simple random walk on $\operatorname{Comb}(\mathbb{Z}, f)$, independent of $X$, and $U_{n}^{\prime}, V_{n}^{\prime}, W_{k}^{\prime}, T_{k}^{\prime}, \theta_{n}^{\prime}$ be the corresponding counterparts. Define another sequence of stopping times by setting $\sigma_{0}=0$ and

$$
\sigma_{m+1}:=\inf \left\{n>\sigma_{m}: U_{n}=U_{n}^{\prime} \text { and }\left(U_{n} \neq U_{n-1} \text { or } U_{n}^{\prime} \neq U_{n-1}^{\prime}\right)\right\} .
$$

Lemma 2.1. For any $\varepsilon>0$, there exists $d \in \mathbb{N}$ such that

$$
\mathbf{P}^{u, v}\left(\sigma_{N} \geq \theta_{d N} \wedge \theta_{d N}^{\prime}\right)<\varepsilon,
$$

for all $N \in \mathbb{N}$ and for all $u, v \in \mathbb{V}_{N}$ with $u_{1}+u_{2}+v_{1}+v_{2}$ being even.

Proof. For $n \geq 0$, define

$$
Z_{2 n}=U_{n}-U_{n}^{\prime} \quad \text { and } \quad Z_{2 n+1}=U_{n+1}-U_{n}^{\prime} .
$$

Define a sequence of stopping times inductively by setting $\tau_{0}=0$ and

$$
\tau_{m}=\inf \left\{n>\tau_{m-1}: Z_{n} \neq Z_{n-1}\right\}
$$


By the strong Markov property, $\left\{Z_{\tau_{m}}, m \geq 0\right\}$ is a simple random walk on $\mathbb{Z}$. Suppose that $u, v \in \mathbb{V}_{N}$. Write $u=\left(u_{1}, u_{2}\right)$ and $v=\left(v_{1}, v_{2}\right)$. Then $\left|u_{1}\right|,\left|v_{1}\right| \leq N$ and

$$
Z_{\tau_{0}}=u_{1}-v_{1} \in[-2 N, 2 N] .
$$

By definition, if $Z_{\tau_{m}}=0$ then

$$
\begin{aligned}
& U_{k}=U_{k}^{\prime}, U_{k} \neq U_{k-1}^{\prime}, \tau_{m}=2 k \text { for some } k \in \mathbb{Z}^{+} \text {; or } \\
& U_{k+1}=U_{k}^{\prime}, U_{k} \neq U_{k}^{\prime}, \tau_{m}=2 k+1 \text { for some } k \in \mathbb{Z}^{+} .
\end{aligned}
$$

Notice that $U_{n}+V_{n}+U_{n}^{\prime}+V_{n}^{\prime}$ is always even under the assumption that $U_{0}+V_{0}+U_{0}^{\prime}+V_{0}^{\prime}=$ $u_{1}+u_{2}+v_{1}+v_{2}$ is even. This fact, together with that $U_{k+1}=U_{k}^{\prime} \neq U_{k}$, implies that $U_{k+1}=U_{k+1}^{\prime}$. For each $M \geq 0$, let

$$
\xi(0, M):=\left|\left\{m: Z_{\tau_{m}}=0,0 \leq m \leq M\right\}\right|,
$$

the local time of 0 by $\left\{Z_{\tau_{m}}, m \geq 0\right\}$. As a result of the previous argument,

$$
\{\xi(0, M) \geq N\} \subseteq\left\{\sigma_{N} \leq \tau_{M}\right\} \subseteq\left\{\sigma_{N} \leq T_{M} \wedge T_{M}^{\prime}\right\}
$$

For each $\widetilde{w} \in \mathbb{Z}$, let $\mathbf{P}^{\widetilde{w}}$ be the probability measure of a simple random walk $\widetilde{W}$ on $\mathbb{Z}$ starting from $\widetilde{w}$. Use $\widetilde{\xi}(\cdot, \cdot)$ to denote the local time by $\widetilde{W}$. By (9.11) of [7], there exists $x \in \mathbb{N}$ such that, for any $\widetilde{w} \in \mathbb{Z},|\widetilde{w}| \leq 2 N$,

$$
\mathbf{P}^{\widetilde{w}}\left(\widetilde{\xi}\left(0, x N^{2}\right) \leq N\right)<\frac{\varepsilon}{2} .
$$

By (2.2), 2.4) and the argument that $\left\{Z_{\tau_{m}}, m \geq 0\right\}$ is a simple random walk on $\mathbb{Z}$, for any $N \in \mathbb{N}$ and $u, v \in \mathbb{V}_{N}$

$$
\mathbf{P}^{u, v}\left(\xi\left(0, x N^{2}\right) \leq N\right)=\mathbf{P}^{u_{1}-v_{1}}\left(\widetilde{\xi}\left(0, x N^{2}\right) \leq N\right)<\frac{\varepsilon}{2} .
$$

Furthermore, by Theorem 2.13 of [7] and the argument that $W$ is a simple random walk on $\mathbb{Z}$, we can find $d \in \mathbb{N}$ such that for any $N \in \mathbb{N}$ and $u \in \mathbb{V}_{N}$,

$$
\mathbf{P}^{u}\left(T_{x N^{2}} \geq \theta_{d N}\right)=\mathbf{P}^{u}\left(\max _{0 \leq k \leq x N^{2}}\left|W_{k}\right| \geq d N\right)=\mathbf{P}^{u_{1}}\left(\max _{0 \leq k \leq x N^{2}}\left|\widetilde{W}_{k}\right| \geq d N\right) \leq \frac{\varepsilon}{4} .
$$

Combining (2.3), 2.5) with (2.6), we arrive at the desired conclusion.

$$
\begin{aligned}
& \mathbf{P}^{u, v}\left(\sigma_{N} \geq \theta_{d N} \wedge \theta_{d N}^{\prime}\right) \\
= & \mathbf{P}^{u, v}\left(\sigma_{N} \geq \theta_{d N} \wedge \theta_{d N}^{\prime}, \sigma_{N} \geq \tau_{x N^{2}}\right)+\mathbf{P}^{u, v}\left(\sigma_{N} \geq \theta_{d N} \wedge \theta_{d N}^{\prime}, \sigma_{N}<\tau_{x N^{2}}\right) \\
\leq & \mathbf{P}^{u, v}\left(\sigma_{N} \geq \tau_{x N^{2}}\right)+\mathbf{P}^{u, v}\left(\sigma_{N} \geq \theta_{d N} \wedge \theta_{d N}^{\prime}, \sigma_{N} \leq T_{x N^{2}} \wedge T_{x N^{2}}^{\prime}\right) \\
\leq & \mathbf{P}^{u, v}\left(\xi\left(0, x N^{2}\right) \leq N\right)+\mathbf{P}^{u, v}\left(\theta_{d N} \leq T_{x N^{2}} \quad \text { or } \quad \theta_{d N}^{\prime} \leq T_{x N^{2}}^{\prime}\right) \\
\leq & \mathbf{P}^{u, v}\left(\xi\left(0, x N^{2}\right) \leq N\right)+\mathbf{P}^{u}\left(\theta_{d N} \leq T_{x N^{2}}\right)+\mathbf{P}^{v}\left(\theta_{d N} \leq T_{x N^{2}}\right) \leq \varepsilon
\end{aligned}
$$

The above lemma shows that, with a small exception, the number of collisions is bounded by departure times $\theta$ and $\theta^{\prime}$ of $U$ and $U^{\prime}$ linearly. In the following applications of Lemma 2.1, $\varepsilon=1 / 2$, we can find $d \in \mathbb{N}$, such that for all $N \in \mathbb{N}$ and for all $u, v \in \mathbb{V}_{N}$ with $u_{1}+u_{2}+v_{1}+v_{2}$ being even,

$$
\mathbf{P}^{u, v}\left(\sigma_{N} \geq \theta_{d N} \wedge \theta_{d N}^{\prime}\right)<\frac{1}{2}
$$


Obviously, $d \geq 2$. We fix such $d$ throughout this section.

Next, we want to estimate the probability that there is at least one collision of $X$ and $X^{\prime}$ once $U$ and $U^{\prime}$ collide. To this end, we define a sequence of events. For $m \geq 0$, let

$$
\begin{aligned}
& \Psi_{m}=\left\{X_{n}=X_{n}^{\prime}, \quad\left|V_{n}\right|+\left|V_{n}^{\prime}\right| \geq\left|V_{\sigma_{m}}\right|+\left|V_{\sigma_{m}}^{\prime}\right|\right. \\
& \text { for some } \left.\sigma_{m} \leq n<\inf \left\{h>\sigma_{m}: V_{h}=0 \text { or } V_{h}^{\prime}=0\right\}\right\} .
\end{aligned}
$$

Notice that $\Psi_{m}$ occurs if $X_{\sigma_{m}}=X_{\sigma_{m}}^{\prime}$. Moreover, suppose that $(u, 0),(u, v) \in \mathbb{V}$ with $v$ being even, if $\Psi_{m} \cap\left\{X_{\sigma_{m}}=(u, 0), X_{\sigma_{m}}^{\prime}=(u, v)\right\}$ occurs then $X$ enters the segment $\{u\} \times[-f(u), f(u)]$ and collides with $X^{\prime}$ at a vertex with height greater than or equal to $v / 2$ after time $\sigma_{m}$ but before $X$ or $X^{\prime}$ exits the segment. Here, by height (of a vertex) we mean the distance from the vertex to the $x$-axis. The next lemma shows that these events have good bounds.

Lemma 2.2. There exist $c_{1}, c_{2}>0$, such that for all $(u, v) \in \mathbb{V}$ with $v$ being even,

$$
\frac{c_{1}}{|v| \vee 1} \leq \mathbf{P}^{(u, 0),(u, v)}\left(\Psi_{0}\right) \leq \frac{c_{2}}{|v| \vee 1} .
$$

Proof. We first examine the case that $f(u) \geq 2 v>0$ and $v$ is even. For $x \in \mathbb{Z}$, define

$$
\tau_{x}=\inf \left\{n>0: X_{n}=(u, x)\right\},
$$

and $\tau_{x}^{\prime}$ similarly. If $\tau_{2 v}<\tau_{0}$ and $X^{\prime}$ stays in $\{u\} \times[v / 2,3 v / 2]$ before time $\tau_{2 v}$, then $X$ and $X^{\prime}$ must collide before time $\tau_{2 v}<\tau_{0} \wedge \tau_{0}^{\prime}$ at a vertex whose height is greater than or equal to $v / 2$. Therefore

$$
\begin{aligned}
& \mathbf{P}^{(u, 0),(u, v)}\left(\Psi_{0}\right) \geq \mathbf{P}^{(u, 0),(u, v)}\left(X_{1}=(u, 1), \tau_{2 v} \leq v^{2} \wedge \tau_{0}, \tau_{v / 2}^{\prime} \vee \tau_{3 v / 2}^{\prime} \geq v^{2}\right) \\
= & \mathbf{P}^{(u, 0)}\left(X_{1}=(u, 1)\right) \mathbf{P}^{(u, 1)}\left(\tau_{2 v} \leq v^{2}, \tau_{2 v}<\tau_{0}\right) \mathbf{P}^{(u, v)}\left(\tau_{v / 2} \vee \tau_{3 v / 2} \geq v^{2}\right) \\
= & \frac{1}{4} \mathbf{P}^{(u, 1)}\left(\tau_{2 v} \leq v^{2}, \tau_{2 v}<\tau_{0}\right) \mathbf{P}^{(u, v)}\left(\tau_{v / 2} \vee \tau_{3 v / 2} \geq v^{2}\right) .
\end{aligned}
$$

Obviously, if $X$ starts from vertex $(u, 1)$ then $X$ will stay at $\{u\} \times[1,2 v-1] \cap \mathbb{Z}^{2}$ before time $\tau_{0} \wedge \tau_{2 v}$. Consider a simple random walk on $\mathbb{Z}$. Let $\left\{\eta_{i}\right\}$ be i.i.d. random variables with $\mathbf{P}\left(\eta_{1}=1\right)=\mathbf{P}\left(\eta_{1}=\right.$ $-1)=1 / 2$, and $S_{k}=1+\sum_{i=1}^{k} \eta_{i}$ for $k \geq 0$. Define stopping times

$$
\tilde{\tau}_{2 v}=\inf \left\{k>0: S_{k} \geq 2 v\right\} \text { and } \tilde{\tau}_{0}=\inf \left\{k>0: S_{k} \leq 0\right\} .
$$

Both $\left\{\tilde{\tau}_{2 v} \leq v^{2}\right\}$ and $\left\{\widetilde{\tau}_{2 v}<\widetilde{\tau}_{0}\right\}$ are increasing events of $\left\{\eta_{i}, i \geq 1\right\}$. By the FKG inequality and the gambler's ruin problem (or refer to Lemma 3.1 of [7]),

$$
\mathbf{P}\left(\tilde{\tau}_{2 v} \leq v^{2}, \tilde{\tau}_{2 v}<\widetilde{\tau}_{0}\right) \geq \mathbf{P}\left(\widetilde{\tau}_{2 v} \leq v^{2}\right) \mathbf{P}\left(\widetilde{\tau}_{2 v}<\widetilde{\tau}_{0}\right)=\mathbf{P}\left(\widetilde{\tau}_{2 v} \leq v^{2}\right) \frac{1}{2 v} .
$$

It can be verified that $\left\{X_{n}: 0 \leq n \leq \tau_{0} \wedge \tau_{2 v}\right\}$ conditioned on $\left\{X_{0}=(u, 1)\right\}$ has the same law as $\left\{S_{n}: 0 \leq n \leq \widetilde{\tau}_{2 v} \wedge \widetilde{\tau}_{0}\right\}$. So

$$
\mathbf{P}^{(u, 1)}\left(\tau_{2 v} \leq v^{2}, \tau_{2 v}<\tau_{0}\right)=\mathbf{P}\left(\tilde{\tau}_{2 v} \leq v^{2}, \widetilde{\tau}_{2 v}<\tilde{\tau}_{0}\right) .
$$

By Theorem 2.13 of [7] again, there exists $c_{1}>0$ independent of $u$ and $v$, such that

$$
\mathbf{P}\left(\tilde{\tau}_{2 v} \leq v^{2}\right) \geq c_{1} \quad \text { and } \quad \mathbf{P}^{(u, v)}\left(\tau_{v / 2} \vee \tau_{3 v / 2} \geq v^{2}\right) \geq c_{1}
$$


Taking (2.8)-(2.11) together, we obtain the first inequality of the lemma.

$$
\mathbf{P}^{(u, 0),(u, v)}\left(\Psi_{0}\right) \geq \frac{c_{1}^{2}}{8 v}
$$

We now turn to the proof of the second inequality. Define

$$
H=\sum_{n=0}^{\infty} 1_{\left\{X_{n}=X_{n}^{\prime}, n<\tau_{0} \wedge \tau_{0}^{\prime}\right\}},
$$

the number of collisions of $X$ and $X^{\prime}$ before one of them exits $\{u\} \times[1, f(u)]$. Then

$$
\begin{aligned}
& \mathbf{E}^{(u, 0),(u, v)}(H)=\sum_{n=0}^{\infty} \sum_{x=1}^{f(u)} \mathbf{P}^{(u, 0),(u, v)}\left(X_{n}=X_{n}^{\prime}=(u, x), n<\tau_{0} \wedge \tau_{0}^{\prime}\right) \\
= & \sum_{n=0}^{\infty} \sum_{x=1}^{f(u)} \mathbf{P}^{(u, 0)}\left(X_{n}=(u, x), n<\tau_{0}\right) \mathbf{P}^{(u, v)}\left(X_{n}=(u, x), n<\tau_{0}\right) \\
\leq & 2 \sum_{n=0}^{\infty} \sum_{x=1}^{f(u)} \mathbf{P}^{(u, 0)}\left(X_{n}=(u, x), n<\tau_{0}\right) \mathbf{P}^{(u, x)}\left(X_{n}=(u, v), n<\tau_{0}\right) \\
= & 2 \sum_{n=0}^{\infty} \mathbf{P}^{(u, 0)}\left(X_{2 n}=(u, v), 2 n<\tau_{0}\right) \\
= & 2 \mathbf{E}^{(u, 0)}(\text { number of visits to }(u, v) \text { by } X \text { before returning to }(u, 0)) .
\end{aligned}
$$

In the previous arguments, the inequality follows from the property of reversible Markov chain and the fact that the reversible measure for $x \in[1, f(u)] \cap \mathbb{Z}$ is just the degree of the vertex. Therefore

$$
\mathbf{P}^{(u, v)}\left(X_{n}=(u, x), n<\tau_{0}\right) \leq 2 \mathbf{P}^{(u, x)}\left(X_{n}=(u, v), n<\tau_{0}\right)
$$

By Theorem 9.7 of [7],

$$
\mathbf{E}^{(u, 0),(u, v)}(H) \leq 2 .
$$

The second inequality of the lemma will follow once we show that there exists $c_{2}>0$ independent of $u, v$ such that

$$
\mathbf{E}^{(u, 0),(u, v)}\left(H \mid \Psi_{0}\right) \geq c_{2} v .
$$

When the event $\Psi_{0}$ occurs, there is a collision at vertex $(u, w)$ for some $w$ with $w \geq v / 2$. Conditioned on this event, the total number of collisions in the set $\{u\} \times[v / 3, f(u)]$ will be greater than the number of collisions that take place before the first time that one of the random walks exits this interval. A lower bound could be obtained by considering two independent simple random walks in an interval, starting at $v / 2$. Before hitting either $v / 3$ or $2 v / 3$, the average number of collisions is the average number of returning to the starting point before exiting the interval, which is exactly the Green function of a simple random walk starting at $v / 2$, before exiting the interval $(v / 3,2 v / 3)$. This is of order $v$.

By 2.13) and 2.14), we conclude that $\mathbf{P}^{(u, 0),(u, v)}\left(\Psi_{0}\right) \leq 2 /\left(c_{2} v\right)$. This completes the proof of the case that $f(u) \geq 2 v>0$ and $v$ is even. The proof can be modified to treat other cases and is omitted here. 
Now we are ready to state a key lemma. To be concise, we set

$$
\breve{f}(n)=1 \vee \max _{-n \leq i \leq n} f(i) .
$$

As a result, $\breve{f}$ is a strictly positive and increasing function on $\mathbb{Z}^{+}$.

Lemma 2.3. There exists $c>0$ such that, for all $N \in \mathbb{N}$ and for all $u, v \in \mathbb{V}_{N}$ with $u_{1}+u_{2}+v_{1}+v_{2}$ being even,

$$
\mathbf{P}^{u, v}\left(X_{n}=X_{n}^{\prime} \text { for some } n \in\left[0, \theta_{d N} \wedge \theta_{d N}^{\prime}\right)\right) \geq \frac{c N}{\breve{f}(d N)+N}
$$

Proof. Let

$$
H=\sum_{m=1}^{N}\left(\left|V_{\sigma_{m}}\right| \vee\left|V_{\sigma_{m}}^{\prime}\right| \vee 1\right) 1_{\Psi_{m}} 1_{\left\{\sigma_{m}<\theta_{d N} \wedge \theta_{d N}^{\prime}\right\}} .
$$

If $H>0$ then $X$ and $X^{\prime}$ collide before time $\theta_{d N} \wedge \theta_{d N}^{\prime}$. We shall use the second moment method to estimate the probability of $\{H>0\}$. Calculate directly as follows.

$$
\begin{aligned}
& \mathbf{E}^{u, v}(H)=\sum_{m=1}^{N} \mathbf{E}^{u, v}\left(\left(\left|V_{\sigma_{m}}\right| \vee\left|V_{\sigma_{m}}^{\prime}\right| \vee 1\right) 1_{\Psi_{m}} 1_{\left\{\sigma_{m}<\theta_{d N} \wedge \theta_{d N}^{\prime}\right\}}\right) \\
= & \sum_{m=1}^{N} \mathbf{E}^{u, v}\left[\mathbf{E}^{u, v}\left(\left(\left|V_{\sigma_{m}}\right| \vee\left|V_{\sigma_{m}}^{\prime}\right| \vee 1\right) 1_{\Psi_{m}} 1_{\left\{\sigma_{m}<\theta_{d N} \wedge \theta_{d N}^{\prime}\right\}} \mid X_{i}, X_{i}^{\prime}, 0 \leq i \leq \sigma_{m}\right)\right] \\
= & \sum_{m=1}^{N} \mathbf{E}^{u, v}\left[\left(\left|V_{\sigma_{m}}\right| \vee\left|V_{\sigma_{m}}^{\prime}\right| \vee 1\right) \mathbf{P}^{u, v}\left(\Psi_{m} \mid X_{i}, X_{i}^{\prime}, 0 \leq i \leq \sigma_{m}\right) ; \sigma_{m}<\theta_{d N} \wedge \theta_{d N}^{\prime}\right] \\
= & \sum_{m=1}^{N} \mathbf{E}^{u, v}\left[\left(\left|V_{\sigma_{m}}\right| \vee\left|V_{\sigma_{m}}^{\prime}\right| \vee 1\right) \mathbf{P}^{X_{\sigma_{m}}, X_{\sigma_{m}}^{\prime}}\left(\Psi_{0}\right) ; \sigma_{m}<\theta_{d N} \wedge \theta_{d N}^{\prime}\right] \\
\geq & \sum_{m=1}^{N} \mathbf{E}^{u, v}\left(c_{1} ; \sigma_{m}<\theta_{d N} \wedge \theta_{d N}^{\prime}\right) \quad \text { by Lemma } 2.2 \\
\geq & c_{1} N \mathbf{P}^{u, v}\left(\sigma_{N}<\theta_{d N} \wedge \theta_{d N}^{\prime}\right) \geq \frac{c_{1}}{2} N \quad \text { by 2.7). }
\end{aligned}
$$

Here the equation (2.15) is by the strong Markov property. Applying Lemma 2.2 and the strong Markov property again, we have the following estimates.

$$
\begin{aligned}
& \mathbf{E}^{u, v}\left(H^{2}\right) \\
= & \mathbf{E}^{u, v}\left(\sum_{m=1}^{N}\left(\left|V_{\sigma_{m}}\right| \vee\left|V_{\sigma_{m}}^{\prime}\right| \vee 1\right) 1_{\Psi_{m}} 1_{\left\{\sigma_{m}<\theta_{d N} \wedge \theta_{d N}^{\prime}\right\}} \sum_{n=1}^{N}\left(\left|V_{\sigma_{n}}\right| \vee\left|V_{\sigma_{n}}^{\prime}\right| \vee 1\right) 1_{\Psi_{n}} 1_{\left\{\sigma_{n}<\theta_{d N} \wedge \theta_{d N}^{\prime}\right\}}\right) \\
= & \mathbf{E}^{u, v}\left(\sum_{m=1}^{N}\left(\left|V_{\sigma_{m}}\right| \vee\left|V_{\sigma_{m}}^{\prime}\right| \vee 1\right)^{2} 1_{\Psi_{m}} 1_{\left\{\sigma_{m}<\theta_{d N} \wedge \theta_{d N}^{\prime}\right\}}\right) \\
& +2 \mathbf{E}^{u, v}\left(\sum_{m=1}^{N}\left(\left|V_{\sigma_{m}}\right| \vee\left|V_{\sigma_{m}}^{\prime}\right| \vee 1\right) 1_{\Psi_{m}} 1_{\left\{\sigma_{m}<\theta_{d N} \wedge \theta_{d N}^{\prime}\right\}} \sum_{n>m}^{N}\left(\left|V_{\sigma_{n}}\right| \vee\left|V_{\sigma_{n}}^{\prime}\right| \vee 1\right) 1_{\Psi_{n}} 1_{\left\{\sigma_{n}<\theta_{d N} \wedge \theta_{d N}^{\prime}\right\}}\right)
\end{aligned}
$$




$$
\begin{aligned}
& \leq 2 \breve{f}(d N) \mathbf{E}^{u, v}\left(\sum_{m=1}^{N}\left(\left|V_{\sigma_{m}}\right| \vee\left|V_{\sigma_{m}}^{\prime}\right| \vee 1\right) 1_{\Psi_{m}} 1_{\left\{\sigma_{m}<\theta_{d N} \wedge \theta_{d N}^{\prime}\right\}}\right) \\
& \quad+2 \sum_{m=1}^{N} \sum_{n>m}^{N} \mathbf{E}^{u, v}\left(\left(\left|V_{\sigma_{m}}\right| \vee\left|V_{\sigma_{m}}^{\prime}\right| \vee 1\right) 1_{\Psi_{m}} 1_{\left\{\sigma_{m}<\theta_{d N} \wedge \theta_{d N}^{\prime}\right\}}\left(\left|V_{\sigma_{n}}\right| \vee\left|V_{\sigma_{n}}^{\prime}\right| \vee 1\right) 1_{\Psi_{n}} 1_{\left\{\sigma_{n}<\theta_{d N} \wedge \theta_{d N}^{\prime}\right\}}\right) \\
& =2 \breve{f}(d N) \mathbf{E}^{u, v}(H)+2 \sum_{m=1}^{N} \sum_{n>m}^{N} \mathbf{E}^{u, v}\left(\left(\left|V_{\sigma_{m}}\right| \vee\left|V_{\sigma_{m}}^{\prime}\right| \vee 1\right) 1_{\Psi_{m}} 1_{\left\{\sigma_{m}<\theta_{d N} \wedge \theta_{d N}^{\prime}\right\}} \times\left(\left|V_{\sigma_{n}}\right| \vee\left|V_{\sigma_{n}}^{\prime}\right| \vee 1\right) \mathbf{P}^{X_{\sigma_{n}}, X_{\sigma_{n}}^{\prime}}\left(\Psi_{0}\right) 1_{\left\{\sigma_{n}<\theta_{d N} \wedge \theta_{d N}^{\prime}\right\}}\right) \\
& \leq 2 \breve{f}(d N) \mathbf{E}^{u, v}(H)+2 c_{2} \sum_{m=1}^{N} \sum_{n>m}^{N} \mathbf{E}^{u, v}\left(\left(\left|V_{\sigma_{m}}\right| \vee\left|V_{\sigma_{m}}^{\prime}\right| \vee 1\right) 1_{\Psi_{m}} 1_{\left\{\sigma_{m}<\theta_{d N} \wedge \theta_{d N}^{\prime}\right\}}\right) \\
& \leq\left(2 \breve{f}(d N)+2 c_{2} N\right) \mathbf{E}^{u, v}(H) .
\end{aligned}
$$

By the Hölder inequality,

$$
\mathbf{P}^{u, v}(H>0) \geq \frac{\left[\mathbf{E}^{u, v}(H)\right]^{2}}{\mathbf{E}^{u, v}\left[H^{2}\right]} \geq \frac{\mathbf{E}^{u, v}(H)}{2 \breve{f}(d N)+2 c_{2} N} \geq \frac{c_{1} N}{4 \breve{f}(d N)+4 c_{2} N} .
$$

We have reached the conclusion of the lemma.

Proof of Theorem 1.1 It suffices to consider the case that two independent simple random walks on $\operatorname{Comb}(\mathbb{Z}, f)$ starting from the same vertex $(0,0)$. Notice that as $X, X^{\prime}$ start from $(0,0)$, almost surely $\theta_{d} \wedge \theta_{d}^{\prime}<\theta_{d^{2}} \wedge \theta_{d^{2}}^{\prime}<\theta_{d^{3}} \wedge \theta_{d^{3}}^{\prime}<\cdots$. Define for $m \geq 1$,

$$
\Upsilon_{m}=\left\{X_{n}=X_{n}^{\prime} \quad \text { for some } n \in\left[\theta_{d^{m}} \wedge \theta_{d^{m}}^{\prime}, \theta_{d^{m+1}} \wedge \theta_{d^{m+1}}^{\prime}\right)\right\}
$$

We write $\mathbf{P}=\mathbf{P}^{(0,0),(0,0)}$ and $t=\theta_{d^{m}} \wedge \theta_{d^{m}}^{\prime}$ for short. By the strong Markov property and Lemma 2.3, there exists $c>0$ such that for all $m \in \mathbb{N}$

$$
\begin{aligned}
& \mathbf{P}\left(\Upsilon_{m} \mid 1_{\Upsilon_{i}}, 1 \leq i<m, X_{n}, X_{n}^{\prime}, n \leq \theta_{d^{m}} \wedge \theta_{d^{m}}^{\prime}\right) \\
= & \mathbf{P}^{X_{t}, X_{t}^{\prime}}\left(X_{n}=X_{n}^{\prime} \text { for some } n \in\left[0, \theta_{d^{m+1}} \wedge \theta_{d^{m+1}}^{\prime}\right)\right) \\
\geq & \frac{c d^{m}}{\breve{f}\left(d^{m+1}\right)+d^{m+1}} .
\end{aligned}
$$

We will show below that (1.1) implies that

$$
\sum_{m=1}^{\infty} \frac{d^{m}}{\breve{f}\left(d^{m}\right)+d^{m}}=\infty .
$$

Consequently, $\mathbf{P}\left(\Upsilon_{m}\right.$ infinitely often $)=1$ by the second Borel-Cantelli Lemma (extended version, Page 237 of [4]). Furthermore,

$$
\mathbf{P}\left(X_{n}=X_{n}^{\prime} \text { infinitely often }\right) \geq \mathbf{P}\left(\Upsilon_{m} \text { infinitely often }\right)=1 .
$$

We now prove 2.16). If $\breve{f}\left(d^{m}\right) \leq d^{m}$ for infinitely many $m$, then it is trivial and we have nothing to do. Otherwise, there exists $m_{0}$ such that $\breve{f}\left(d^{m}\right)>d^{m}$ for all $m \geq m_{0}$. Hence it suffices to prove

$$
\sum_{m=1}^{\infty} \frac{d^{m}}{\breve{f}\left(d^{m}\right)}=\infty \text {. }
$$


Set $D_{m}=d+d^{2}+\cdots+d^{m}$. Then $d^{m} \leq D_{m} \leq d^{m+1}$, and

$$
\frac{d^{m}}{\breve{f}\left(d^{m}\right)} \geq \frac{1}{d} \sum_{l=D_{m}+1}^{D_{m+1}} \frac{1}{\breve{f}(l)} \text {. }
$$

So

$$
\sum_{m=1}^{\infty} \frac{d^{m}}{\breve{f}\left(d^{m}\right)} \geq \frac{1}{d} \sum_{m=1}^{\infty} \sum_{l=D_{m}+1}^{D_{m+1}} \frac{1}{\breve{f}(l)}=\frac{1}{d} \sum_{l=d+1}^{\infty} \frac{1}{\breve{f}(l)}=\infty .
$$

Hence (2.16) holds in either case and the proof of the theorem is completed.

\section{Proof of Theorem 1.4}

In this section we will show a slightly more general result from which Theorem 1.4 will follow.

Theorem 3.1. If $\sum_{i=-n}^{n} f(i)=O(n)$, then $\operatorname{Comb}(\mathbb{Z}, f)$ has the infinitely many triple collisions property.

To prove the theorem we introduce yet another independent simple random walk $X^{\prime \prime}$ on $\operatorname{Comb}(\mathbb{Z}, f)$. For any $u, v, w \in \mathbb{V}$, we write $\mathbf{P}^{u, v, w}$ for the joint probability measure of the three independent simple random walks $X, X^{\prime}$ and $X^{\prime \prime}$ starting from $u, v$ and $w$, respectively. Let $\mathbf{E}^{u, v, w}$ be the corresponding expectation. By the assumption of Theorem 3.1, there exists $c>4$, such that for all $n \in \mathbb{N}$,

$$
\sum_{i=-n}^{n} f(i) \leq\left(\frac{c}{2}-2\right) n \text {. }
$$

Hence we fix $f$ and $c$ which satisfy (3.1) throughout this section. Since

$$
([-n, n] \times\{0\}) \cap \mathbb{Z}^{2} \subseteq \mathbb{V}_{n}=\left(\bigcup_{i=-n}^{n}\{i\} \times[-f(i), f(i)]\right) \cap \mathbb{Z}^{2}
$$

therefore

$$
2 n \leq\left|\mathbb{V}_{n}\right| \leq c n
$$

Recall that $\theta_{n}$ is defined in (2.1).

Lemma 3.2. For any $\varepsilon>0$, there exists $d \in \mathbb{N} \backslash\{1\}$ such that $\mathbf{P}^{u}\left(\theta_{d n} \leq n^{2}\right) \leq \varepsilon$ for all $n \in \mathbb{N}$ and for all $u \in \mathbb{V}_{n}$.

Proof. For each $\widetilde{w} \in \mathbb{Z}$, let $\mathbf{P}^{\widetilde{w}}$ be the probability measure of a simple random walk $\widetilde{W}$ on $\mathbb{Z}$ starting from $\widetilde{w}$. By Theorem 2.13 of [7] and the argument that $W$ is a simple random walk on $\mathbb{Z}$, there exists $d \in \mathbb{N} \backslash\{1\}$ which depends only on $\varepsilon$, such that for all $n \in \mathbb{N}$ and for all $u \in \mathbb{V}_{n}$

$$
\begin{aligned}
\mathbf{P}^{u}\left(\theta_{d n} \leq n^{2}\right) & \leq \mathbf{P}^{u}\left(\max _{0 \leq k \leq n^{2}}\left|W_{k}\right| \geq d n\right)=\mathbf{P}^{u_{1}}\left(\max _{0 \leq k \leq n^{2}}\left|\widetilde{W}_{k}\right| \geq d n\right) \\
& \leq \mathbf{P}^{0}\left(\max _{0 \leq k \leq n^{2}}\left|\widetilde{W}_{k}\right| \geq(d-1) n\right) \leq \varepsilon .
\end{aligned}
$$

For $x \in \mathbb{V}$, let $\tau_{x}=\inf \left\{m \geq 0: X_{m}=x\right\}$ be the hitting time of $x$ by $X$. 
Lemma 3.3. For $\varepsilon>0$, there exists $c_{1} \in \mathbb{N}$ such that $\mathbf{P}^{u}\left(\tau_{v}>c_{1} n^{2}\right) \leq \varepsilon$ for all $n \in \mathbb{N}$ and for all $u, v \in \mathbb{V}_{n}$.

Proof. Fix $0<\varepsilon<1, n \in \mathbb{N}$ and $u \in \mathbb{V}_{n}$. Let $h, c_{1} \in \mathbb{N}$ such that

$$
h>\frac{8(c+1)}{\varepsilon} \text { and } c_{1}>\frac{6 c^{2} h^{2}}{\varepsilon} .
$$

Suppose that the random walk $X$ starts from vertex $u \in \mathbb{V}_{n}$. Since $\theta_{h n}=\tau_{(h n, 0)} \wedge \tau_{(-h n, 0)}$ for $u \in \mathbb{V}_{n}$, then

$$
\begin{aligned}
\mathbf{P}^{u}\left(\tau_{v}>c_{1} n^{2}\right) & \leq \mathbf{P}^{u}\left(\tau_{v}>\theta_{h n}\right)+\mathbf{P}^{u}\left(\theta_{h n}>c_{1} n^{2}\right) \\
& \leq \mathbf{P}^{u}\left(\tau_{v}>\tau_{(h n, 0)}\right)+\mathbf{P}^{u}\left(\tau_{v}>\tau_{(-h n, 0)}\right)+\mathbf{P}^{u}\left(\theta_{h n}>c_{1} n^{2}\right)
\end{aligned}
$$

Hence we are going to estimate probabilities of the three events above.

Because $\operatorname{Comb}(\mathbb{Z}, f)$ is a tree, there exists a unique simple path from $v$ to $(h n, 0)$. If $u$ is a vertex on the path, then by the gambler's ruin problem and the fact that $X$ is a recurrent process,

$$
\mathbf{P}^{u}\left(\tau_{v}>\tau_{(h n, 0)}\right)=\frac{\operatorname{dist}(u, v)}{\operatorname{dist}(v,(h n, 0))}
$$

where $\operatorname{dist}(\cdot, \cdot)$ is the graph distance. By the assumption (3.3),

$$
\mathbf{P}^{u}\left(\tau_{v}>\tau_{(h n, 0)}\right) \leq \frac{2 n+\max _{-n \leq k \leq n} f(k)}{h n-n} \leq \frac{2+c}{h-1}<\frac{\varepsilon}{3} .
$$

If $u$ is not on the path, then with probability one $X$ will reach the path first at $v,\left(v_{1}, 0\right)$ or $\left(u_{1}, 0\right)$. A slight change in the proof actually shows that (3.5) holds for these cases. So in all cases, (3.5) gives an upper bound of the probability of the first event. Similarly, the probability of the second event is also small.

$$
\mathbf{P}^{u}\left(\tau_{v}>\tau_{(-h n, 0)}\right)<\frac{\varepsilon}{3}
$$

We now study the third event. Let $\tilde{\mathbb{G}}$ be the spanning tree of $\mathbb{V}_{h n}$, which is a connected subgraph of $\operatorname{Comb}(\mathbb{Z}, f)$. The process $X$ before time $\theta_{h n}$ is also a simple random walk on $\tilde{G}$. Regard $\widetilde{G}$ as an electric network that each edge is assigned a unit conductance. Then by Proposition 10.6 of [6],

$$
\mathbf{E}^{u}\left(\theta_{h n}\right) \leq c_{\widetilde{G}} \Re(u \leftrightarrow\{-h n, h n\}),
$$

where $c_{\widetilde{G}}$ is twice of the total conductance and $\Re(u \leftrightarrow\{-h n, h n\})$ is the effective resistance between $u$ and $\{-h n, h n\}$. Since $\widetilde{\mathbb{G}}$ is a tree, we can easily get that

$$
c_{\widetilde{G}}=2\left|\mathbb{V}_{h n}\right|-2 \quad \text { and } \quad \Re(u \leftrightarrow\{-h n, h n\}) \leq\left|\mathbb{V}_{h n}\right| .
$$

Consequently, $\mathbf{E}^{u}\left(\theta_{h n}\right) \leq 2\left|\mathbb{V}_{h n}\right|^{2} \leq 2 c^{2} h^{2} n^{2}$. By the Markov inequality and 3.3 , we get the probability of the third event

$$
\mathbf{P}^{u}\left(\theta_{h n}>c_{1} n^{2}\right) \leq \frac{\varepsilon}{3} .
$$

Combining (3.4)-(3.7) together we complete the proof of the lemma. 
By Lemmas 3.2 and 3.3, we can find $c_{1} \in \mathbb{N}$ and $d \in \mathbb{N} \backslash\{1\}$ such that

$$
\mathbf{P}^{u}\left(\tau_{v}>c_{1} n^{2}\right) \leq \frac{1}{4} \text { and } \mathbf{P}^{u}\left(\theta_{d n} \leq c_{1} n^{2}\right) \leq \frac{1}{4}
$$

for all $n \in \mathbb{N}$ and for all $u, v \in \mathbb{V}_{n}$. Fix such $c_{1}$ and $d$ throughout this section. We are now ready to proceed to a similar key argument as Lemma 2.3 .

Lemma 3.4. There exists $c_{2}>0$ such that for all $N \in \mathbb{N} \backslash\{1\}$ and for all $u, v, w \in \mathbb{V}_{N}$ with $(-1)^{u_{1}+u_{2}}=$ $(-1)^{v_{1}+v_{2}}=(-1)^{w_{1}+w_{2}}$,

$$
\mathbf{P}^{u, v, w}\left(X_{n}=X_{n}^{\prime}=X_{n}^{\prime \prime} \text { for some } n \in[0, \Theta)\right) \geq \frac{c_{2}}{\log N},
$$

where $\Theta=\theta_{d N} \wedge \theta_{d N}^{\prime} \wedge \theta_{d N}^{\prime \prime}=\inf \left\{m \geq 0:\left\{X_{m}, X_{m}^{\prime}, X_{m}^{\prime \prime}\right\} \nsubseteq \mathbb{V}_{d N-1}\right\}$

Proof. Fix $N \in \mathbb{N} \backslash\{1\}$ and $u, v, w \in \mathbb{V}_{N}$. Notice that $\Theta$ is the first time that one of $X, X^{\prime}, X^{\prime \prime}$ exits $\mathrm{V}_{d N-1}$. Let

$$
H=\sum_{n=0}^{2 c_{1} N^{2}} 1_{\left\{X_{n}=X_{n}^{\prime}=X_{n}^{\prime \prime} \in \mathbb{V}_{N}, n<\Theta\right\}}
$$

We will prove that

$$
\mathbf{E}^{u, v, w}(H) \geq c_{1}^{*} \quad \text { and } \quad \mathbf{E}^{u, v, w}\left(H^{2}\right) \leq c_{2}^{*} \mathbf{E}^{u, v, w}(H) \log N .
$$

Then by the Hölder inequality,

$$
\mathbf{P}^{u, v, w}\left(X_{n}=X_{n}^{\prime}=X_{n}^{\prime \prime} \text { for some } n<\Theta\right) \geq \mathbf{P}^{u, v, w}(H>0) \geq \frac{\left[\mathbf{E}^{u, v, w}(H)\right]^{2}}{\mathbf{E}^{u, v, w}\left(H^{2}\right)} \geq \frac{c_{1}^{*}}{c_{2}^{*} \log N}
$$

Now we begin to prove (3.9). For $x \in \mathbb{V}_{N}$, let $q_{n}(u, x)=\mathbf{P}^{u}\left(X_{n}=x, \theta_{d N}>n\right)$. Then $q_{2 n}(x, x)$ is decreasing in $n$ by the spectral theory (or referring to [1]). By the strong Markov property, for any $c_{1} N^{2} \leq n \leq 2 c_{1} N^{2}$ with $u_{1}+u_{2}+x_{1}+x_{2}+n$ and $k+n$ being even,

$$
\begin{aligned}
& \mathbf{P}^{u}\left(X_{n}=x, \theta_{d N}>n\right)=\sum_{k=0}^{n} \mathbf{P}^{u}\left(X_{n}=x, \tau_{x}=k, n<\theta_{d N}\right) \\
= & \sum_{k=0}^{n} \mathbf{P}^{u}\left(\tau_{x}=k<\theta_{d N}\right) \mathbf{P}^{u}\left(X_{n}=x, n<\theta_{d N} \mid \tau_{x}=k<\theta_{d N}\right) \\
= & \sum_{k=0}^{n} \mathbf{P}^{u}\left(\tau_{x}=k<\theta_{d N}\right) q_{n-k}(x, x) \\
\geq & \sum_{k=0}^{n} \mathbf{P}^{u}\left(\tau_{x}=k<\theta_{d N}\right) q_{2 c_{1} N^{2}}(x, x) \\
= & q_{2 c_{1} N^{2}}(x, x) \mathbf{P}^{u}\left(\tau_{x} \leq n, \theta_{d N}>\tau_{x}\right) \\
\geq & q_{2 c_{1} N^{2}}(x, x)\left(\mathbf{P}^{u}\left(\tau_{x} \leq c_{1} N^{2}\right)+\mathbf{P}^{u}\left(\theta_{d N}>c_{1} N^{2}\right)-1\right)
\end{aligned}
$$




$$
\geq \frac{1}{2} q_{2 c_{1} N^{2}}(x, x) \quad \text { by }(3.8) \text {. }
$$

As a result,

$$
\begin{aligned}
& \mathbf{E}^{u, v, w}(H)=\sum_{x \in \mathbb{V}_{N}} \sum_{n=0}^{2 c_{1} N^{2}} \mathbf{P}^{u, v, w}\left(X_{n}=X_{n}^{\prime}=X_{n}^{\prime \prime}=x, \Theta>n\right) \\
& =\sum_{x \in \mathbb{V}_{N}} \sum_{n=0}^{2 c_{1} N^{2}} \mathbf{P}^{u}\left(X_{n}=x, \theta_{d N}>n\right) \mathbf{P}^{v}\left(X_{n}=x, \theta_{d N}>n\right) \mathbf{P}^{w}\left(X_{n}=x, \theta_{d N}>n\right) \\
& \geq \frac{1}{8} \sum_{x \in \mathbb{V}_{N}} \sum_{\substack{c_{1} N^{2} \leq n \leq 2 c_{1} N^{2} \\
u_{1}+u_{2}+x_{1}+x_{2}+n \text { even }}}\left[q_{2 c_{1} N^{2}}(x, x)\right]^{3} \\
& \geq \frac{c_{1} N^{2}}{16} \sum_{x \in \mathbb{V}_{N}}\left[q_{2 c_{1} N^{2}}(x, x)\right]^{3} \\
& \geq \frac{c_{1} N^{2}}{16\left|\mathbb{V}_{N}\right|^{2}}\left[\sum_{x \in \mathbb{V}_{N}} q_{2 c_{1} N^{2}}(x, x)\right]^{3} \geq \frac{c_{1}}{16 c^{2}}\left[\sum_{x \in \mathbb{V}_{N}} q_{2 c_{1} N^{2}}(x, x)\right]^{3} .
\end{aligned}
$$

The third inequality is by the Hölder inequality. Applying the Hölder inequality and (3.8) again, we have the following estimates.

$$
\begin{aligned}
\sum_{x \in \mathbb{V}_{N}} q_{2 c_{1} N^{2}}(x, x) & \geq \sum_{x \in \mathbb{V}_{N}} \sum_{y \in \mathbb{V}_{d N}} q_{c_{1} N^{2}}(x, y) q_{c_{1} N^{2}}(y, x) \\
& \geq \frac{1}{4} \sum_{x \in \mathbb{V}_{N}} \sum_{y \in \mathbb{V}_{d N}}\left[q_{c_{1} N^{2}}(x, y)\right]^{2} \\
& \geq \frac{1}{4\left|\mathbb{V}_{d N}\right|} \sum_{x \in \mathbb{V}_{N}}\left(\sum_{y \in \mathbb{V}_{d N}} q_{c_{1} N^{2}}(x, y)\right)^{2} \\
& \geq \frac{\left|\mathbb{V}_{N}\right|}{4\left|\mathbb{V}_{d N}\right|} \min _{x \in \mathbb{V}_{N}}\left[\sum_{y} \mathbf{P}^{x}\left(X_{c_{1} N^{2}}=y, \theta_{d N}>c_{1} N^{2}\right)\right]^{2} \\
& \geq \frac{1}{2 c d} \min _{x \in \mathbb{V}_{N}}\left[\mathbf{P}^{x}\left(\theta_{d N}>c_{1} N^{2}\right)\right]^{2} \geq \frac{1}{4 c d} .
\end{aligned}
$$

Combining the two estimates together, we obtain that

$$
\mathbf{E}^{u, v, w}(H) \geq \frac{c_{1}}{16 c^{2}} \cdot\left(\frac{1}{4 c d}\right)^{3}=\frac{c_{1}}{1024 c^{5} d^{3}} .
$$

The first part of 3.9 is proved by choosing $c_{1}^{*}=c_{1} c^{-5} d^{-3} / 1024$.

We now turn to the second moment, i.e., the second part of $(3.9)$. Since that $\operatorname{Comb}(\mathbb{Z}, f)$ is a graph with uniformly bounded degree, by Corollary 14.6 of [8], there exists $c_{0}^{*}>0$ such that $\mathbf{P}^{x}\left(X_{k}=\right.$ $y) \leq c_{0}^{*} / \sqrt{k}$ for all $x, y \in \mathbb{V}$. Hence,

$$
\mathbf{P}^{x, x, x}\left(X_{k}=X_{k}^{\prime}=X_{k}^{\prime \prime}\right)=\sum_{y \in \mathbb{V}} \mathbf{P}^{x, x, x}\left(X_{k}=X_{k}^{\prime}=X_{k}^{\prime \prime}=y\right)
$$




$$
=\sum_{y \in \mathbb{V}}\left[\mathbf{P}^{x}\left(X_{k}=y\right)\right]^{3} \leq \frac{\left(c_{0}^{*}\right)^{2}}{k} \sum_{y \in \mathbb{V}} \mathbf{P}^{x}\left(X_{k}=y\right)=\frac{\left(c_{0}^{*}\right)^{2}}{k} .
$$

By the inequality above and the strong Markov property,

$$
\begin{aligned}
& \mathbf{E}^{u, v, w}\left(H^{2}\right)=\mathbf{E}^{u, v, w}\left(\sum_{n=0}^{2 c_{1} N^{2}} \sum_{x \in \mathbb{V}_{N}} 1_{\left\{X_{n}=X_{n}^{\prime}=X_{n}^{\prime \prime}=x, \Theta>n\right\}} \sum_{k=0}^{2 c_{1} N^{2}} \sum_{y \in \mathbb{V}_{N}} 1_{\left\{X_{k}=X_{k}^{\prime}=X_{k}^{\prime \prime}=y, \Theta>k\right\}}\right) \\
& \leq \mathbf{E}^{u, v, w}(H)+2 \mathbf{E}^{u, v, w}\left(\sum_{n=0}^{2 c_{1} N^{2}} \sum_{x \in \mathbb{V}_{N}} 1_{\left\{X_{n}=X_{n}^{\prime}=X_{n}^{\prime \prime}=x, \Theta>n\right\}} \sum_{k>n}^{2 c_{1} N^{2}} 1_{\left\{X_{k}=X_{k}^{\prime}=X_{k}^{\prime \prime}\right\}}\right) \\
& =\mathbf{E}^{u, v, w}(H)+2 \sum_{n=0}^{2 c_{1} N^{2}} \sum_{x \in \mathbb{V}_{N}} \sum_{k>n}^{2 c_{1} N^{2}} \mathbf{P}^{u, v, w}\left(X_{n}=X_{n}^{\prime}=X_{n}^{\prime \prime}=x, \Theta>n, X_{k}=X_{k}^{\prime}=X_{k}^{\prime \prime}\right) \\
& =\mathrm{E}^{u, v, w}(H) \\
& +2 \sum_{n=0}^{2 c_{1} N^{2}} \sum_{x \in \mathbb{V}_{N}} \sum_{k>n}^{2 c_{1} N^{2}} \mathbf{P}^{u, v, w}\left(X_{n}=X_{n}^{\prime}=X_{n}^{\prime \prime}=x, \Theta>n\right) \mathbf{P}^{x, x, x}\left(X_{k-n}=X_{k-n}^{\prime}=X_{k-n}^{\prime \prime}\right) \\
& \leq \mathrm{E}^{u, v, w}(H) \\
& +2 \sum_{n=0}^{2 c_{1} N^{2}} \sum_{x \in \mathbb{V}_{N}} \mathbf{P}^{u, v, w}\left(X_{n}=X_{n}^{\prime}=X_{n}^{\prime \prime}=x, \Theta>n\right) \sum_{k=1}^{2 c_{1} N^{2}} \mathbf{P}^{x, x, x}\left(X_{k}=X_{k}^{\prime}=X_{k}^{\prime \prime}\right) \\
& \leq \mathbf{E}^{u, v, w}(H)\left(1+2 \max _{x \in \mathbb{V}_{N}} \sum_{k=1}^{2 c_{1} N^{2}} \mathbf{P}^{x, x, x}\left(X_{k}=X_{k}^{\prime}=X_{k}^{\prime \prime}\right)\right) \\
& \leq \mathrm{E}^{u, v, w}(H)\left(1+2 \max _{x \in \mathbb{V}_{N}} \sum_{k=1}^{2 c_{1} N^{2}} \frac{\left(c_{0}^{*}\right)^{2}}{k}\right) \\
& \leq\left(1+2\left(c_{0}^{*}\right)^{2} \log \left(2 c_{1}\right)+4\left(c_{0}^{*}\right)^{2} \log N\right) \mathbf{E}^{u, v, w}(H) \text {. }
\end{aligned}
$$

We have thus verified the second part of (3.9) and finished the proof.

Proof of Theorem 3.1 It suffices to consider the case that three independent simple random walks on $\operatorname{Comb}(\mathbb{Z}, f)$ starting from the same vertex $(0,0)$. For each $m \geq 1$, denote by $\Upsilon_{m}$ the event

$$
\left\{X_{n}=X_{n}^{\prime}=X_{n}^{\prime \prime} \quad \text { for some } n \in\left[\theta_{d^{m}} \wedge \theta_{d^{m}}^{\prime} \wedge \theta_{d^{m}}^{\prime \prime}, \theta_{d^{m+1}} \wedge \theta_{d^{m+1}}^{\prime} \wedge \theta_{d^{m+1}}^{\prime \prime}\right)\right\} \text {. }
$$

Then by the strong Markov property and Lemma 3.4 there exists $c_{2}>0$ such that

$$
\begin{aligned}
& \mathbf{P}\left(\Upsilon_{m} \mid 1_{\Upsilon_{i}}, 1 \leq i<m, X_{n}, X_{n}^{\prime}, X_{n}^{\prime \prime}, n \leq \theta_{d^{m}} \wedge \theta_{d^{m}}^{\prime} \wedge \theta_{d^{m}}^{\prime \prime}\right) \\
= & \mathbf{P}_{t}^{X_{t}, X_{t}^{\prime}, X_{t}^{\prime \prime}}\left(X_{n}=X_{n}^{\prime}=X_{n}^{\prime \prime} \text { for some } n \in\left[\theta_{d^{m}} \wedge \theta_{d^{m}}^{\prime} \wedge \theta_{d^{m}}^{\prime \prime}, \theta_{d^{m+1}} \wedge \theta_{d^{m+1}}^{\prime} \wedge \theta_{d^{m+1}}^{\prime \prime}\right)\right) \\
\geq & \frac{c_{2}}{\log d^{m}}=\frac{c_{2}}{m \log d},
\end{aligned}
$$


where $\mathbf{P}=\mathbf{P}^{(0,0),(0,0),(0,0)}$ and $t=\theta_{d^{m}} \wedge \theta_{d^{m}}^{\prime} \wedge \theta_{d^{m}}^{\prime \prime}$. By the second Borel-Cantelli Lemma, $\mathbf{P}\left(\Upsilon_{m}\right.$ occurs infinitely often) $=1$. Furthermore,

$$
\mathbf{P}\left(X_{n}=X_{n}^{\prime}=X_{n}^{\prime \prime} \text { infinitely often }\right) \geq \mathbf{P}\left(\Upsilon_{m} \text { infinitely often }\right)=1
$$

This completes the proof of Theorem 3.1 .

\section{A Related Result}

The proof of Theorem 1.3 is almost the same as the case $f(x)=x^{\alpha}$ for $1<\alpha<2$ in [1]. So we just outline the changes needed to run the proof. Fix $\beta>0$ and $f(x)=|x| \log ^{\beta}(|x| \vee 1)$ for $x \in \mathbb{Z}$.

Lemma 4.1. Let $k \geq 2$ and $y=(k, h) \in \mathbb{V}$. Let $t \in \mathbb{N}, s \in \mathbb{R}$ with $s^{3} \log ^{\beta} s=t$. Then there exists $c>0$ such that the transition density $q$ of the simple random walk on $\operatorname{Comb}(\mathbb{Z}, f)$ satisfies:

$$
q_{t}(0, y) \leq \begin{cases}c s^{-2}(\log s)^{-\beta} & \text { if } s \geq k, \\ c k^{-2}(\log k)^{-\beta} & \text { if } s<k .\end{cases}
$$

Proof. Following the proof of Lemma 5.1 of [1], we consider the simple random walk $X$ starting from $(0,0)$. The first coordinate makes roughly $k^{2}$ steps to go from $k / 2$ to $k$. At each step of the horizontal coordinate we perform an independent experiment. We say the experiment is successful if we spend time greater than $k^{2} \log ^{2 \beta} k$ in the vertical line segment in this step of the first coordinate. The probability of success is then bounded below by $c_{1} /\left(k \log ^{\beta} k\right)$ and in the $k^{2}$ experiments with high probability there will be a success and the expected number of successes is $k(\log k)^{-\beta}$, thus the total time needed to reach $k$ will be of order $k^{3} \log ^{\beta} k$. We omit the details of the proof here.

Assume that $1 \leq h \leq k \log ^{\beta} k$. Let $Q_{k, h}=\{(k, w): 0 \leq w \leq h\}$, and $Z_{k, h}$ be the number of collisions of the two random walks in $Q_{k, h}$. We also define $\widetilde{Z}_{k, h}=Z_{k, 2 h / 3}-Z_{k, h / 3}$ to be the number of collisions that happen in the set $\widetilde{Q}_{k, h}=\{(k, w): h / 3 \leq w \leq 2 h / 3\}$.

Lemma 4.2. There exist $c^{\prime}, c^{\prime \prime}>0$ such that for each $k \geq 2$ and $1 \leq h \leq k \log ^{\beta} k, \mathbf{E} Z_{k, h} \leq$ $c^{\prime} h /\left(k \log ^{\beta} k\right) ; \quad \mathbf{E}\left(Z_{k, h} \mid \widetilde{Z}_{k, h}>0\right) \geq c^{\prime \prime} h$.

Proof. Let $s=g(t)$ be the inverse function of $t=s^{3} \log ^{\beta} s$. By Lemma 4.1 .

$$
\begin{aligned}
\mathbf{E} Z_{k, h} & =\sum_{t} \sum_{x \in Q_{k, h}} q_{t}(0, x)^{2} \leq \sum_{t<k^{3} \log ^{\beta} k} \frac{c^{2} h}{k^{4} \log ^{2 \beta} k}+\sum_{t \geq k^{3} \log ^{\beta} k} \frac{c^{2} h}{s^{4} \log ^{2 \beta} s} \\
& \leq \frac{c^{2} h}{k \log ^{\beta} k}+c^{2} h \int_{k^{3} \log ^{\beta} k}^{\infty} \frac{1}{(g(t))^{4} \log ^{2 \beta} g(t)} d t \\
& =\frac{c^{2} h}{k \log ^{\beta} k}+c^{2} h \int_{k}^{\infty} \frac{1}{s^{4} \log ^{2 \beta} s} d\left(s^{3} \log ^{\beta} s\right) \\
& =\frac{c^{2} h}{k \log ^{\beta} k}+c^{2} h \int_{k}^{\infty} \frac{3}{s^{2} \log ^{\beta} s} d s+c^{2} h \int_{k}^{\infty} \frac{\beta}{s^{2} \log ^{\beta+1} s} d s \leq \frac{c^{\prime} h}{k \log ^{\beta} k} .
\end{aligned}
$$


The second inequality is proved similarly as Lemma 5.2 of [1].

Proof of Theorem 1.3. By Lemma 4.2. $\mathbf{P}\left(\widetilde{Z}_{k, h}>0\right) \leq \frac{c^{\prime}}{c^{\prime \prime} k \log ^{\beta} k}$ for each $k \geq 2$ and $1 \leq h \leq k \log ^{\beta} k$. Summing over all $k \geq 2$ and over all $h$ ranging over powers of 2 and satisfying $1 \leq h \leq k \log ^{\beta} k$, we get that

$$
\sum_{k} \sum_{h \text { power of } 2} \mathbf{P}\left(\widetilde{Z}_{k, h}>0\right) \leq \frac{c^{\prime}}{c^{\prime \prime}} \sum_{k} \frac{\log _{2}\left(k \log ^{\beta} k\right)}{k \log ^{\beta} k}<\infty \text {, since } \beta>2 \text {. }
$$

Hence the total number of sets $\widetilde{Q}_{k, h}$ with at least one collision is almost surely finite. Since $\mathbf{E}\left(\widetilde{Z}_{k, h}\right) \leq \mathbf{E}\left(Z_{k, h}\right)<\infty$, the number of collisions in each set $\widetilde{Q}_{k, h}$ is a.s. finite. So, the total number of collisions in $\{(k, w) \in \mathbb{V}: k \geq 2, w \geq 1\} \subseteq \bigcup_{k, h} \widetilde{Q}_{k, h}$ is a.s. finite. We omit the details of the rest of the proof here.

Acknowledgments. We are grateful to the anonymous referees for their careful reading and the suggestion that shortens the proof of Lemma 3.3 .

\section{References}

[1] Barlow, M.T., Peres, Y., Sousi, P., Collisions of Random Walks, http://arxiv.org/PS_cache/arxiv/ $\mathrm{pdf} / 1003 / 1003.3255 \mathrm{v} 1 . \mathrm{pdf}$.

[2] Chen, X., Chen, D., Two random walks on the open cluster of $\mathbb{Z}^{2}$ meet infinitely often. Science China Mathematics, 53, 1971-1978 (2010). MR2679079

[3] Chen. D., Wei, B. and Zhang, F.,A note on the finite collision property of random walks. Statistics and Probability Letters, 78, 1742-1747, (2008). MR2453913

[4] Durrett, R. Probability: Theory and Examples, 3rd ed. Brooks/Cole, Belmont, 2005. MR1068527

[5] Krishnapur, M. and Peres,Y., Recurrent graphs where two independent random walks collide finitely often. Elect. Comm. in Probab. 9, 72-81, (2004). MR2081461

[6] Levin, D.A., Peres, Y. and Wilmer, E. L., Markov Chains and Mixing Times, American Mathematical Society, Providence, RI, 2008. MR2466937

[7] Révész, P., Random walk in random and non-random environments. 2nd edition, World Scientific Publishing Co., New Jersey, 2005. MR2168855

[8] Woess, W., Random walks on Infinite Graph and Groups. Cambridge University Press, Cambridge,UK, 2000. MR1743100 